\title{
RollCall : The Design For A Low-Cost And Power Efficient Active RFID Asset Tracking System
}

\author{
Gautam D. Bhanage*, Yu Zhang*, Yanyong Zhang*, Wade Trappe*, Richard. E. Howard ${ }^{\dagger}$ \\ *WINLAB, Rutgers University, Piscataway, NJ, USA, e-mail: gautamb,yu,yyzhang,trappe@winlab.rutgers.edu \\ †PNP Networks, Los Altos, CA, USA, e-mail: reh@att.net
}

\begin{abstract}
One of the most compelling and immediate applications of pervasive computing would be to use RF technology to support low-cost, long-lived and continual tracking of assets. Unfortunately, initial solutions have not yet led to widespread deployment. We believe that meeting the economic and system requirements of this application requires a redesign of the tag, the transmission protocol, and the algorithms used by basestations to identify tags, all with the underlying goal of reducing cost and power consumption through simplification. In this paper, we propose a new inventory tracking system, called RollCall, in which a transmit-only RFID tag will be attached to every item, and these tags will report their presence to the readers periodically by broadcasting the tag IDs so that a missing tag/item can be quickly identified. The power conservation obtained from short transmissions on a very simple MAC layer combined with the hardware cost and size reduction from having a simple radio stack on the tag provides considerable economic, dimensional and tag lifetime benefits. In this paper, we present the design and architecture of the RollCall system, and conduct preliminary studies to examine the feasibility of building such a system by tweaking offthe-shelf signal processing algorithms. Initial studies and simulation results suggest that it is possible to monitor about 5000 tags in a store with networked basestations at a low error rate with an extended tag life time of at least a year based on conservative estimates with non-custom tag radio and micro-controllers.
\end{abstract}

Keywords-Active RFID, Asset tracking, ROLLCALL.

\section{INTRODUCTION}

\section{A. RFID Technology \& Asset Tracking}

Pervasive asset monitoring with RFID technology requires regular polling of the tags attached to the goods. A wide range of RFID tags are commercially available, ranging from battery powered active tags to backscatter based passive tags. Passive tags could provide an economically viable solution to asset tracking, but are not suitable for pervasive monitoring applications as they would necessitate high-power basestations that cannot be deployed due to possible regulatory challenges. To overcome the close reading range requirements, it is thus necessary to use active tags. Active tags face a major design tradeoff between the transmission range, batterylife and the size of a tag. Sophisticated designs have led to considerably large tags (size $>15 \mathrm{~cm}$ ) and inflated prices (tens of dollars)[2]. Various experiments with MAC protocols such as CSMA[3], TDMA[4] and 802.11[5] on tags have failed to address the high cost and power consumption issues at the tag.

\section{B. System Design Considerations}

The infrastructure and the tag cost are the parameters that decide the expense of an asset tracking system. We are considering a typical system with a large number of tags (thousands) and comparatively small number of basestations (tens), and therefore the overall system cost is influenced more by the cost at the tag than at the reader.

Parameters dictating cost and power savings at the tag include:

- Hardware Architecture: Previous studies done in [6] show that the amount of power consumed in short range radios for idle listening, receiving and sending a packet are comparable. [7] shows that the MICA2 motes from CrossBow operating at $433 \mathrm{MHz}$ and $0 \mathrm{dBm}$ have the power consumption ratio for idlelistening: receiving: transmitting as $1: 1: 1.41$. These studies show receiver hardware as a significant power sink.

- Protocol Stack: Limited hardware capabilities mandate the use of simple MAC protocols which should achieve significant cost and power savings. Various isolated approaches to a simple protocol stack (like using an 802.11 based tag sending almost a 50Byte beacon[8]) without significant simplification of the hardware itself may result in power savings on the radio but will still incur some cost for the tag hardware itself.

Based on the above observations we propose the deployment of an asymmetric system design. Our approach is to make an extremely simple tag by completely eliminating the receiver and the channel sensing capabilities and having a simple MAC on the tag. An asymmetric link between the tag and the basestation presents significant cost and power savings for the tags but correspondingly increases the complexity of tag detection at the basestation. We are currently designing an active tag inventory monitoring system, which we call RollCall. This involves a reconsideration of everything from the tag hardware to the simplification of tag transmission protocols and the specification of basestation algorithms. However, this paper is focused on issues related to the redesign of the transmission and reception protocols associated with a 


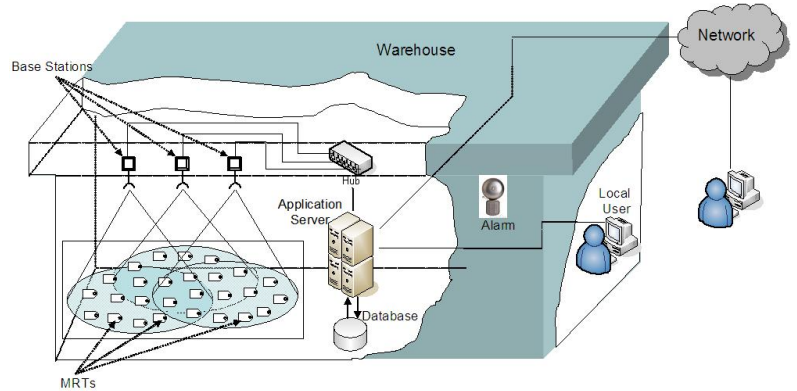

Fig. 1. Architecture diagram for the RollCall inventory management system.

transmit only active tag and its base station, and their relation to a robust asset tracking system. Our tag hardware design will be described in a subsequent follow-up work as the actual system is deployed.

The rest of the paper is organized as follows. In Section II, we provide the architecture of the RollCall system. Section III begins with a feasibility study which establishes the need for good detection schemes. The detection algorithms mentioned in Section IV attempt to achieve tag detection under severe operating conditions. Section V shows the performance under varying operating conditions. Conclusions and future directions for exploration are given by section VI.

\section{Overview of The RollCall System}

Figure 1 illustrates a sample deployment of the proposed RollCall system. The deployment is usually hosted in an authorized area where all the valuables carry a Micro Radio Tag (MRT, also referred to as tag throughout this paper). MRTs, which are on-off keying (OOK) based transmission-only tags that emit radio signals periodically to announce the presence of the corresponding items. OOK was chosen (over better SNR approaches like BPSK) as the modulation scheme due to its simple and efficient hardware realization. An array of basestations (i.e. tag readers) are deployed with sufficient density so that each MRT would be in the coverage area of at least two basestations as long as it stays in the confined area. The processing at the basestation could be performed on-board (if the reader has a sufficiently powerful DSP/FPGA which provides a fairly cheap solution), or can be performed off-board by a PC (e.g. samples may be transferred via PCI Express). Movement of an MRT will cause it to come closer to different basestations and thus trigger a "handoff" process that would facilitate tag tracking. These readers are usually connected to a central application that supports a real-time display and tracking of items. Existing approaches to real time asset tracking in the recent years have seen increasing size and cost of the tags for this functionality. An asymmetric design should allow for a low cost and small tag size implementation with our system.

The radio link between the tag and the basestation is the most critical tier in the RollCall design and is studied in detail by considering a single-reader multipletag scenario. Each tag transmits its unique ID at a random start time, once in every epoch. For prototyping we are using an epoch duration ( $\mathrm{T}$ ) of 1 second. If a tag is not heard for a few epochs, the reader will announce it missing. Conventionally such a design would imply poor performance. However, unlike other systems that support arbitrary communications, our design requires the MRTs to transmit only one packet ever their ID packet. Beaconing the ID with a transmit-only tag not only eliminates all MAC layer complexities associated with channel sensing, synchronization and reception on the tag but also facilitates a cheaper hardware design allowing lower system costs. In this paper, we set out to investigate the issues such as collisions due to random transmissions, and the feasibility of the system using offthe-shelf signal processing algorithms for detecting the tags from a received signal (which may be corrupted).

Problem Formulation For Each Basestation: The RollCall approach thus requires the problem to be solved at two levels of abstraction: at the basestation level, and at the application level. To evaluate the problem to be solved at the basestation level, let us consider a scenario with a single basestation in the vicinity of multiple MRTs. Each MRT $i$ transmits its tag ID, $C_{i}(t)$ in every epoch. Since the tags are randomly scattered spatially, they have varying distances from the basestation. For the simplified model, the free space path loss model gives the relationship between the received signal strength $P_{r}$ at the basestation and the transmitted power $P_{t}^{i}$ for tag $i$ as: $P_{r}=\left(\frac{\sqrt{G_{l}} \lambda}{4 \pi d}\right)^{2} P_{t}^{i}=\frac{a}{d^{2}} P_{t}^{i}$, where $G_{l}, \pi$, and $\lambda$ are constants, and $d$ is the distance between the transmitter and the receiver. In this the value of the term $a$ is constant and is given by $\left(\frac{\sqrt{G_{l}} \lambda}{4 \pi}\right)^{2}$. For MRT $i$, let $d_{i}$ denote its distance to the reader and $\tau_{i}$ denote its transmission time in the first epoch. Assuming a uniform propagation environment, and considering $T>>L$, where $L$ is a tag's transmission time, the received signal from the MRT $T_{i}$ is given by:

$$
r_{i}(t)=\sum_{n=0}^{\infty} \frac{a}{d_{i}^{2}} C_{i}\left(t-n T-\tau_{i}\right) .
$$

Thus, the complete received baseband signal for all $m$ tags including noise seen by reader $j$ is:

$$
R_{j}(t)=\sum_{i=1}^{m} r_{i}(t)+n(t) .
$$

The detection schemes described in the following sections will be required to isolate the noise $n(t)$ and determine the presence of each of the $C_{i}(t)$ from the $R_{j}(t)$ seen at each basestation $j$.

System-Wide Problem Definition: If $P_{j}$ is the set of the tags that are seen by basestation $j$ and $H_{j}$ is the set of tags that are not seen by the base station $j$ despite being in $j$ 's range, such that $P_{j} \cup H_{j}=\Re_{j}^{b, k}$ where $\Re_{j}^{b, k}$ is the set of $k$-bit tag ID's in the basestation $j$ 's range then, each 


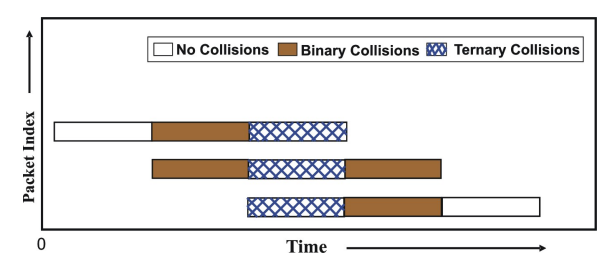

Fig. 2. An illustration of a 3-way collision with two embedded 2-way collisions

basestation $j$ is essentially solving an assignment problem $\Delta_{j}$ given by:

$$
\Delta_{j}:\left\{R_{j}(t: 0 \rightarrow T) \times \Re_{j}^{b, k}\right\} \longrightarrow\left\{P_{j} \cup H_{j}\right\}
$$

For all the $q$ basestations in the system, the set of tags that are not seen may be defined at any instant as $H_{\text {sys }}=H_{1} \cup$ $H_{2} \cup \ldots \cup H_{q}$ and those that are present may be similarly defined as $P_{\text {sys }}=P_{1} \cup P_{2} \cup \ldots \cup P_{q}$. Based on these sets, it is required that every tag missed by a baseststation should be covered by at least one other basestation which may be specified as:

$$
\forall h_{j} \in H_{\text {sys }}, \quad \exists p_{k} \mid\left(p_{k} \in P_{\text {sys }} \cap h_{j}=p_{k}\right)
$$

Since the focus of this paper is on detecting tags at the base station, our discussion assumes there is only one reader in the system in the sections to follow.

\section{Collisions In A Receiver-Less TAG Design}

The definition of the problem in the previous section establishes the need for regular packet detection at the receiver, to keep packet loss at a minimum. Though ALOHA[9] has a similar design and relies on random transmissions, the throughput seen from an ALOHA system is different from our RollCall scenario. Evaluation of the ALOHA protocol in [10] provides an upper bound on the throughput achievable in random transmission systems. However, such a model assumes that the traffic is Poisson i.e, each source is Poisson, which does not reflect our traffic pattern where each source transmits once per epoch. Our analysis aims to quantify the amount of collisions that such a system would experience and shows that the number is non-trivial.

We define a general $r$-way collision to be a collision in which $r$ transmissions overlap with each other for at least one bit. For example, the scenario illustrated in Figure 2 consists of a ternary collision $(r=3)$ and two binary collisions $(r=2)$.

\section{A. Estimation For Packet Level Collisions}

First, let us break an epoch into $N$ slots, each slot corresponding to the time taken to transmit one bit, and let us assume that one tag transmission occupies $k$ contiguous slots. The probability of a slot being occupied by a tag is thus defined as $\alpha=\frac{k}{N}$.

To simplify our discussion, let us initially assume that our system has 2-way collisions only, following which we will generalize the discussion to include higher order collisions. Let the discrete random variable $X_{i}$ denote whether tag $i$ has binary collision(s). $X_{i}$ is set to one if $i$ has binary collision(s) and zero otherwise. Hence, with $m$ tags per basestation, the total number of corrupted packets is given by the random variable $X=\sum_{i=1}^{m} X_{i}$. The total number of expected binary collisions $\left(\psi_{2}\right)$ is evaluated as:

$$
\psi_{2}=E(X)=\sum_{i=1}^{m} E\left(X_{i}\right)=m E\left(X_{i}\right)=m P_{t a g}
$$

where $P_{t a g}$ is the probability of a tag colliding with another tag. Let us assume that the transmission from tag $i$ occupies slots from $a$ to $a+k-1$. Then any tag $j$ with start time $\delta_{j} \in[a-k+1, a+k-1]$ will collide with tag $i$. The probability of tag $j$ having such a start time is given by Bernoulli's trials where a tag having a binary collision is defined as a success event and anything else is a failure.

$$
P_{\text {tag }}=\left(\begin{array}{c}
m-1 \\
1
\end{array}\right)(2 \alpha)^{1}(1-2 \alpha)^{m-2} .
$$

Hence, we have

$$
\psi_{2} \approx m^{2}(2 \alpha)(1-2 \alpha)^{m-2} \approx m^{2}(2 \alpha)
$$

Generalizing the above equation we can calculate the expected number of packets that are involved in an $r$-way collision as:

$$
\psi_{r} \approx m^{r}(2 \alpha)^{r-1}(1-2 \alpha)^{m-r} .
$$

The above analysis gives an estimate for the number of colliding packets in the system. However, we believe that $\psi$ can only partially reflect the system performance because the reader may still be able to extract the sender tag ID from a corrupted packet if only a small portion of that packet is corrupted. Following the length convention, a tag ID usually has 96 or 128 bits, which should provide enough redundancy so that a collided tag may still be detected based on the un-garbled portion. This suggests that along with the number of collided packets, the average number of bits corrupted per packet is another important metric for determining the effect of collisions.

\section{B. Estimation For Bit Level Collisions}

Consider the discrete random variable $X_{i}(1 \leq i \leq N)$ which represents whether the $i$-th bit in an epoch is occupied by two or more tags: $X_{i}=1$ when the bit is occupied by two or more tags, and $X_{i}=0$ otherwise. Hence, the total number of corrupted bits are given by the random variable $X=\sum_{i=1}^{N} X_{i}$. To estimate the number of bits that are corrupted due to binary collisions $\left(\gamma_{2}\right)$, we will first determine the number of bits that are occupied by 2 or more packets $E(X)$, and the number of bits that are occupied by 3 or more packets $E(Y)$, and then subtract the latter from the former, i.e. $\gamma_{2}=E(X)-E(Y)$. $E(X)$ can be evaluated as the sum of individual expected values for each $E\left(X_{i}\right)$. Each $E\left(X_{i}\right)$ is determined by the probability of bit $i$ having at least two packets. If $\alpha$ is the probability of a tag occupying bit $i$, then $\alpha^{2}$ is the probability of two tags using the same bit $i$. Therefore, 


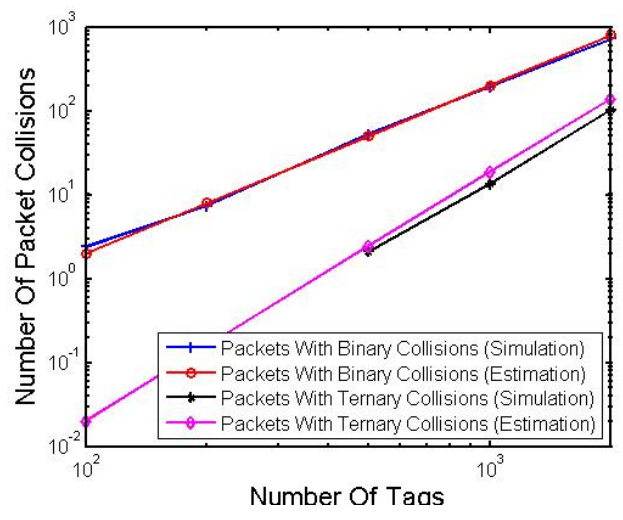

Fig. 3. Packet level collision statistics as a function of number of tags

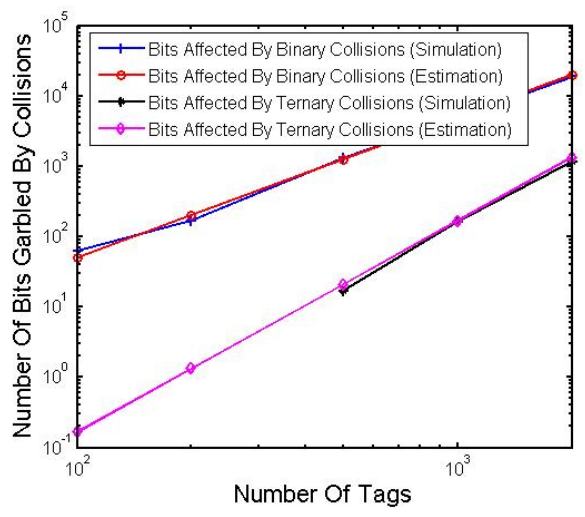

Fig. 4. Bit level collision statistics as a function of number of tags

the expected value for having a collision over slot $i$ is defined as: $E\left(X_{i}\right)=\left(\begin{array}{c}m \\ 2\end{array}\right) \alpha^{2}$. Hence, we have:

$$
E(X)=\frac{m^{2} k^{2}}{2 N}, E(Y)=\frac{m^{3} k^{3}}{6 N^{2}} .
$$

As a result, $\gamma_{2}$ is given by:

$$
\gamma_{2}=\left(\frac{m^{2} k^{2}}{2}-\frac{m^{3} k^{3}}{6 N}\right) \frac{1}{N} .
$$

Generalizing the analysis, the number of bits corrupted by an $\mathrm{r}$-way collision $(2<r<m-1)$ is given as

$$
\gamma_{r}=\left(\frac{(m k)^{r}}{r !}-\frac{(m k)^{r+1}}{(r+1) ! N}\right) \frac{1}{N^{r-1}} .
$$

\section{Model Validation By Simulations}

After deriving these two metrics $\left(\gamma_{r}, \psi_{r}\right)$, we set up simulations to validate our analysis. The results in Figure 3 and Figure 4 show that these two types of results agree with each other. Both results show that the number of packets (and bits) affected by $r$-way collisions grow as the $r$-th power of the number of tags until higher $(r+1$ and above) level collisions start dominating.

Using the models we have developed, we can calculate the number of corrupted packets. Consider that we have 1000 tags with 100 bit IDs, and that these tags report their IDs once per second. The tag data rate of $1 \mathrm{Mbps}$ gives us a slot width of $1 \mu \mathrm{sec}$ and $10^{6}$ slots for every epoch. Based on the model, the number of packets corrupted with a binary collision is approximately 200 . The number of bits corrupted by binary collisions is 5000 . Unfortunately, the error rate under these conditions is intolerable, compared to our desired error rate. As a result, we need to employ signal processing schemes at the receiver to improve detection rate despite the practical problems shown here.

\section{Signal Processing Techniques to Reduce DETECTION ERRORS}

Signal processing is required to separate colliding tag transmissions. Any such scheme requires a good design at both the transmitter and receiver side. For the transmitter side, our approach is to make the tag IDs as orthogonal as possible and exploit this orthogonality in the receiver design to achieve tolerable read failure rates.

\section{A. Tag ID Design \& Transmission}

With simplicity as an essential we chose On-Off keying (OOK) encoding. Thus, tag design relies on generating orthogonal unipolar binary bit vectors. The simplest way to do this (for a large number of 100-bit long vectors) is to use random binary bit vectors. Any random binary tag ID code set at the basestation $d$ given by $\Re_{d}^{b, L}$ will satisfy the auto $\left(A_{j}\right)$ and cross $\left(X_{j k}\right)$ correlation bounds $(\alpha, \beta)$ given in equation (12) and equation (13). These bounds hold for some arbitrary $\alpha$ and $\beta$ for all tags $C_{k}(t)$ and $C_{j}(t)$ in $\Re_{d}^{b, L} . x_{i}$ and $y_{i}$ are the values of two random variables that indicate the expected value of the bit at the position $i$ in the random binary bit vectors $C_{j}$ and $C_{k}$ respectively, and $L$ is the length of the bit vectors in $\Re_{d}^{b, L}$. If $\Re_{d}^{b, L}$ has the characteristic that $\alpha>>\beta$, then the $\Re_{d}^{b, L}$ is considered fairly orthogonal.

$$
\begin{gathered}
\int_{0}^{L} C_{j}(t) \cdot C_{k}(t) d t=E\left(\sum_{i=1}^{L} x_{i}\right)=A_{j} \geq \alpha \\
\int_{0}^{L} C_{j}(t) \cdot C_{k}(t) d t=E\left(\sum_{i=1}^{L} x_{i} y_{i}\right)=X_{j k} \leq \beta
\end{gathered}
$$

The average values of parameters $A_{j}$ and $X_{j k}$ are given by: $A_{j}=L / 2, \quad X_{j k}=L / 4$. The average value of $A_{j}$ is determined by the weight of the code and is justified by the fact that on average half the bits of a random vector will be 1 . The average value of $X_{j k}$ is half that of $A_{j}$ because on average half the 1's from two random bit vectors $j$ and $k$ will align. Based on the average values of $A_{j}$ and $X_{j k},\{\alpha, \beta\}$ can be controlled to generate an optimal codeset with random binary vectors.

\section{B. The Receiver Side: Signal Detection Techniques}

Evaluation of the operating conditions reveals that the problem we are trying to solve is similar to the multiuser detection (MUD) problem [11] in CDMA systems. However, some crucial differences may be sighted as: 1) No Spread Spectrum Transmission: Our approach will not use a spread spectrum type of transmission scheme like that used in CDMA, instead it will just send each symbol in the tag ID as it is, 2) Lack of Power Control: 
Since the MRTs do not have a receiver on them, they cannot control their transmission power to alleviate the near-far problem and 3) Transmitter Modulation: Our simple MRTs will use a low power OOK modulation scheme as compared to the BPSK used in conventional CDMA systems. Initially assume that the transmissions from different MRTs constructively add in phase. This can be achieved by aligning the local oscillators of the tags using some reference phase detection mechanism. Later, in the simulations we explain the working of a completely asynchronous system.

The most naive detection scheme relies on checking the correlation value across the received signal sequence for each tag ID in the codeset $\Re_{d}^{b, L}$ at each basestation $d$. As indicated by (12), the correlation of a tag ID with the received signal from a complete epoch will yield a peak when its transmission is encountered. Such a scheme would declare that the tag is present at the position of the highest correlation value if the correlation index is above a certain pre-set threshold.

Though this simple technique has been widely used as an underlying approach in CDMA, it will fare poorly in our system. Lack of power control schemes and start time estimations with this approach make it prone to detection errors due to collisions and varying signal attenuation effects.

In order to alleviate the adverse impact of this near-far problem, we propose the use of two enhanced techniques; one involves the use of derivatives to locate a tag's presence more accurately while the other is a successive interference cancelation approach. The former technique is much simpler, and requires lesser computation, but the latter delivers a considerably better performance at a higher computation cost.

Derivative Enhanced Correlation Based Detection: Autocorrelation of the tag ID causes a very narrow spike at the actual location of the tag, which exhibits a great discontinuity from its vicinity. Though the amplitude of this spike may not always be the highest across all the correlation sequence, the width is distinctive. The jump in the correlation value is almost twice that from its immediate vicinity and may be attributed to the auto and cross correlation values respectively with random tag IDs. Detection of this characteristic slot-wide correlation spike may be obtained by using the peak of the signal obtained by differentiating the correlation signal in discrete time. Further analysis and experiments also show that higher order derivatives like the second order may be able to pull out this spike where a simple first order differentiation fails. However, there is a bound on the highest order of the derivative that can be used and is determined by the errors that are introduced due to increasing residues. A threshold can be set up to minimize the false positives.

Successive Cancellation Based Detection: The derivative-enhanced correlation technique cannot correctly extract a tag ID if the tag's transmission collides with the transmission of those that are spatially closer to the reader. To resolve this problem, successive cancellation correlates the received signal with each of the tag IDs in the codeset $\Re_{d}^{b, L}$ at reader $d$. Using these correlations, we can find the loudest tag and estimate its signal strength. Then we subtract the contribution of this tag. By repeating this process, a relatively small contribution of the weak tags can eventually stand out among the residue of the received signal. This approach is referred to as successive interference cancellation (SIC) and is widely deployed in CDMA based multiuser detection (MUD) systems. Some relevant discussion may be found in [13].

The key component of this algorithm is the estimation of the received signal strength from each individual tag. Due to the use of binary bit vectors with unipolar encoding the conventional estimation schemes based on correlations shown in [14] cannot be applied directly. Lack of synchronization with tags makes this estimation problem harder to solve. Our detection scheme relies on the correlations between the received signal and all the tag IDs in the pseudo orthogonal codeset to estimate the amplitude of the transmission. After we detect the transmission time of the loudest tag using correlations, we produce an estimate of the signal strength by considering the contribution of all the other tags to the correlation value. All existing CDMA based detection schemes rely on some form of power control mechanism to alleviate the near-far problem. Since power control is absent in MRTs, our algorithm can only use estimated tag signal strength for detection.

Despite the lack of synchrony, power control and the absence of efficient phase estimation schemes, we have tried to tweak the performance of SIC approach to achieve tolerable error rates. Fortunately, though SIC algorithms are sensitive to estimation errors, which is a big concern in our system, theory suggests that as long as the estimation errors remain in reasonable bounds the system can still detect unique signals correctly.

\section{Results And FeAsibility Discussion}

Behavior of the RollCall system (at each basestation) is characterized by the performance of its detection algorithms. Specifically, we considered three detection strategies: the correlation scheme with first and second order derivatives and the SIC scheme. The metric used is tag error rate, i.e. the ratio of the number of tags that were not detected to the total number of tags in that particular reader's range. We model a low interference retail store environment, where tags are located on a plane, and the basestation grid is located in a plane above the tag plane.

It is not practical to do a full RF simulation RollCall. Instead, a more realistic alternative is to characterize the system in baseband with the consideration of three important parameters: 1) carrier phase shift $(\phi)$, which is the difference in the carrier phase of each of the tags with reference to the carrier phase of the first tag in the epoch, 2) time shift $(\delta)$, which is the difference in tag transmission times with reference to the start of the epoch, 


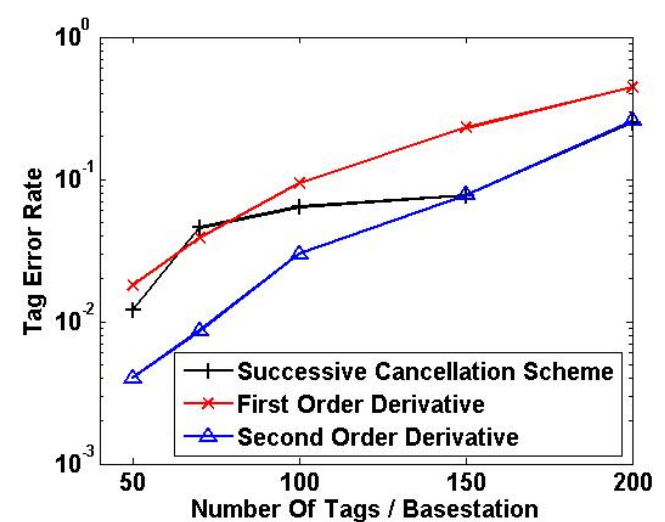

Fig. 5. Tag error rate as a function of the number of tags $(T=10 \mathrm{~ms})$ (a) $\phi=0$

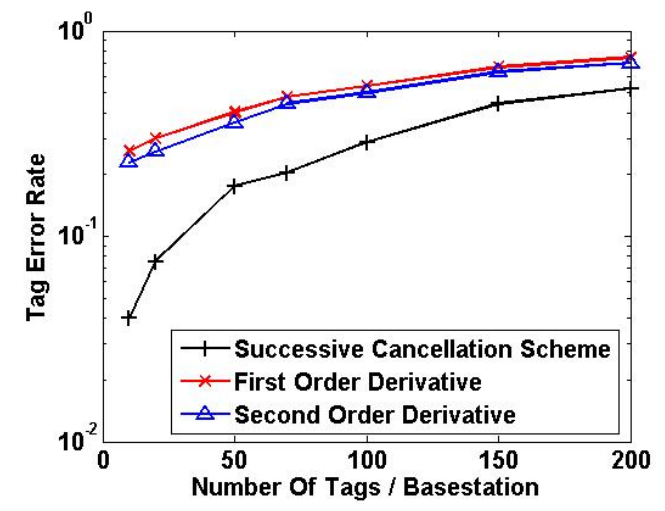

Fig. 6. Tag error rate as a function of the number of tags $(T=10 \mathrm{~ms})$ (b) $\phi=$ random.

and 3) frequency shift $(\Omega)$, which is the difference in the local oscillator frequencies with reference to the specified operating frequency. In the simulations we used random values for $\delta$, and 0 for $\Omega$ because the frequency differences seen with crystal based oscillators were very small. We used 2 settings: (i) $\phi=0$ representing a synchronized setting, which can be achieved by using a PLL, and (ii) $\phi=a$, a uniformly random number between 0 and $2 \pi$, which represents an asynchronous setting where a noncoherent detection of the received signal is required. The effect of multipath is not considered here as we assume that there is no direct obstruction between the tags and the reader. We further assume that a tag's transmission range is sufficiently uniform and independent of tag orientation.

\section{A. Number of Tags Per Basestation}

We first look at impact of the number of tags per basestation. In order to show the effect of interference between the tags, we adopted a rather short epoch duration of 10 milliseconds (or, $10 \mathrm{~ms}$ ) and varied the number of tags from 10 to 200. In this set of experiments, all tags were kept at the same distance from the reader. The results from this test are presented in Figures 7 (synchronous setting) and 6 (asynchronous setting).

In the synchronized setting (shown in Figure 7), all approaches can achieve a decent error rate with a reason-

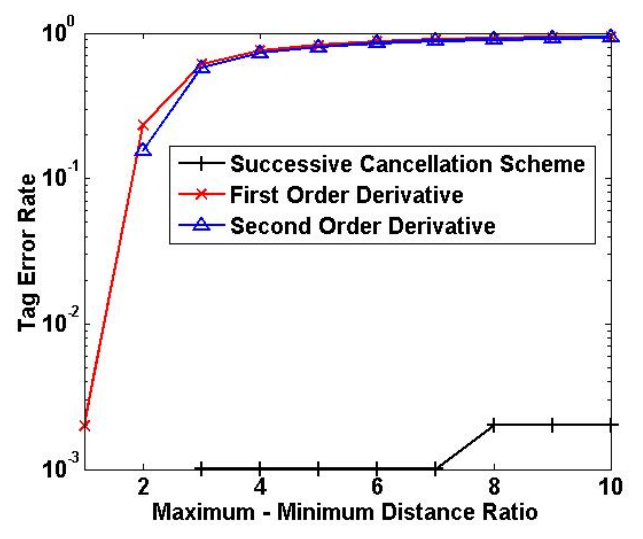

Fig. 7. Tag error rate as a function of $d_{\max } / d_{\min }$, which is an indication of the severity of the near-far problem: (a) $\phi=0$

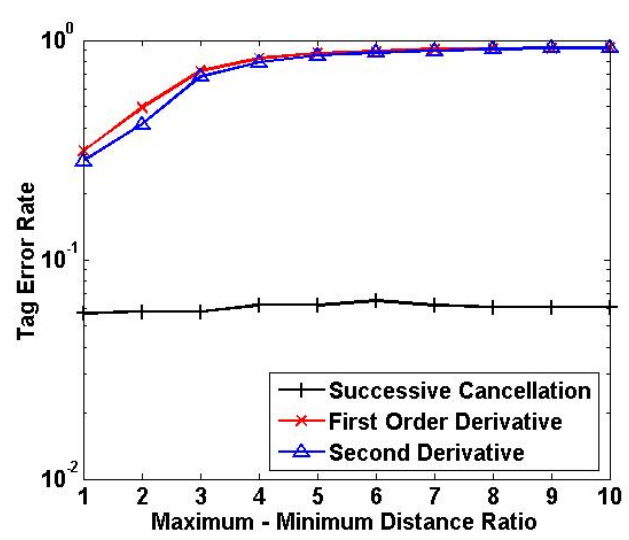

Fig. 8. Tag error rate as a function of $d_{\max } / d_{\min }$, which is an indication of the severity of the near-far problem:(b) $\phi=$ random

ably large number of tags. For instance, the second order derivative and the SIC approach can achieve $1 \%$ error rate when they have 50 tags per $10 \mathrm{~ms}$. We note that the scenario shown in this plot is not very realistic, and it only serves as a basis for comparison. In a more realistic asynchronous setting (shown in Figure 6), the error rate goes up significantly. Due to excessive collisions, the two derivative-enhanced correlation schemes have a poor detection accuracy even with 10 tags, and the SIC scheme can achieve an error rate of $5 \%$ with 20 tags.

Due to these results, in the following experiments we will keep the number of tags small, i.e. 100 tags per 100 ms which corresponds to 10 tags per $10 \mathrm{~ms}$, as we will introduce other factors that further complicate the task of detecting tags.

\section{B. The Impact of Distance Variance}

As discussed in Section IV, the near-far problem has a big impact on the system detection capability. We attempt to quantify the amount of difference in the detection accuracy with the variation in the tag's distance from the basestation. We use the maximum-minimum distance ratio $\left(d_{\max } / d_{\min }\right)$ to represent the ratio of the distance of the tag closest to the basestation to the one which is the farthest. In the interest of simulation complexity, we 


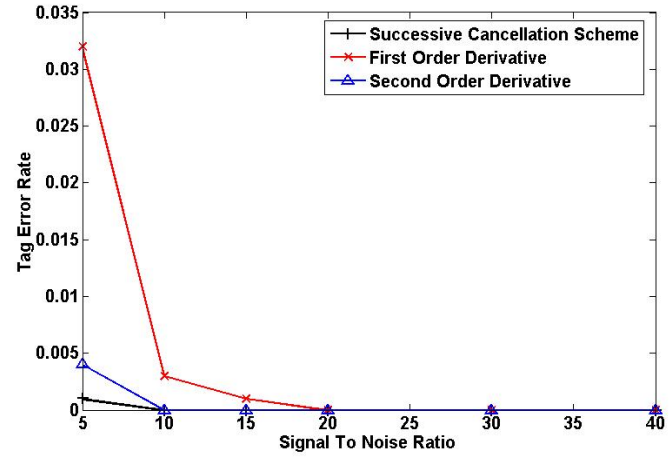

Fig. 9. Tag error rate as a function of SNR (dB): (a) $\phi=0$

adopted a rather short epoch duration of 100 milliseconds (ms) and varied the value of $d_{\max } / d_{\min }$ from 1 to 10 with 100 tags in the environment.

The results are presented in Figures 7 (synchronous setting) and 8 (asynchronous setting). In both settings, the derivative-enhanced correlation techniques degrade rapidly with the increase in the value of $d_{\max } / d_{\min }$ due to the masking of the feeble detection spike by proximal loud tags. The SIC technique is rather robust against the near-far problem. In a synchronous environment the SIC approach has a near perfect performance due to accurate estimations. However, the performance degrades in the asynchronous setting due to the lack of accurate phase estimation techniques to an average error rate of $5 \%$ for 100 tags per $100 \mathrm{~ms}$.

\section{The Impact of SNR On Signal Detection}

Figures 9 and 10 present the tag error rates under varying levels of signal to noise ratio (SNR). In all the simulations, we had 100 tags that reported their ID's within an epoch of 100 milliseconds and $d_{\max } / d_{\min }=1$. With the presence of noise, a tag is considered correctly detected if the estimated transmission start time is located within 10 slots from the actual start time.

The results follow our intuition that as the SNR gets better the detection rates improve. In both figures, we observe a sharp increase in tag error rates after the SNR value drops below a certain level. In a synchronous setting, all three algorithms do well when SNR is above a certain threshold. In an asynchronous setting, the derivative enhanced techniques fail completely. Though the SIC approach performs better, the performance when SNR is below $20 \mathrm{~dB}$ is still poor. In section V-D, we will discuss how to alleviate this problem.

\section{Putting Together a Feasible System}

The previous set of simulations highlighted that SIC could keep the error rate within $10 \%$ in a single epoch. However, we note that this detection accuracy is poor and we should improve the performance using other optimization techniques. For instance, in a real system, we may be able to wait for a few epochs before reporting a missing tag, or we may be able to afford to have a network of basestations so that each basestation is responsible for

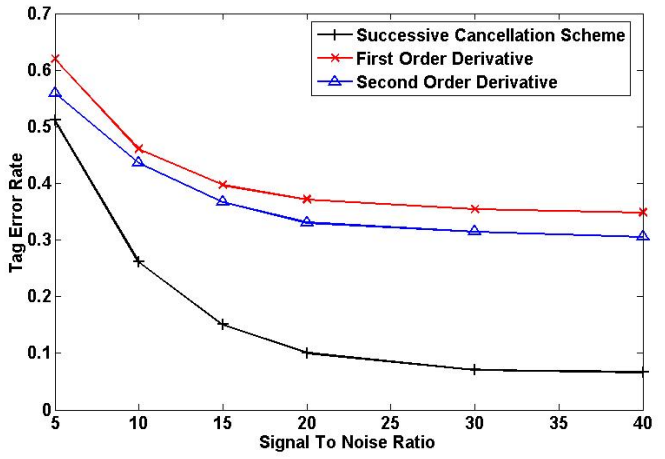

Fig. 10. Tag error rate as a function of SNR (dB): (b) $\phi=$ random

a smaller number of tags. In the interest of space, we only present the result for the SIC scheme since it is the best.

Multiple Epochs: Here, detection is done across multiple runs by setting a simple analog timer on the MRTs that allows the start time of each tag to vary independently within $30 \%$ of its period across multiple epochs. This randomization causes a different set of tags to collide across consecutive epochs. Our simulation results show that we can detect 100 tags with an average error rate less than or equal to $1 \%$ over an interval of 5 epochs (of 100 $\mathrm{ms}$ each). We configured the simulation to represent an environment where we had a $15 \mathrm{~dB}$ SNR and $d_{\max } / d_{\min }$ of 3 . Based on these results, we can safely say that the algorithm would easily scale to at least 1000 tags every 5 seconds (lower bound with a lesser fill factor for the interval) with the same accuracy.

Networked Basestations: Simultaneous detection of a tag's presence across more than one basestation will help to alleviate the near far effect and hence improve detection rate. Simulations done across two basestations show an average improvement in detection accuracy by at least a factor of 3. Integrated simulations done by doing detection across five rounds of $100 \mathrm{msecs}$ each with the tags being covered by at least two basestations, and 100 tags/basestation, at $15 \mathrm{~dB} \mathrm{SNR}$, a $d_{\max } / d_{\min }$ of 3 shows that the error rates are negligible.

\section{E. System Considerations in Deploying RollCall ${ }^{T M}$}

In this section, we discuss the feasibility of deploying RollCall by taking into account the related system issues.

Deployment Scenario: A jewelery store is an ideal place to deploy the RollCall system because the continuous tracking of considerable number of small items is critically important to such places. Let us consider a typical jewelery store with the size of $100 \mathrm{~m}^{2}$ and approximately 5000 items. We need to have a sufficient number of basestations and carefully position them to ensure that each basestation monitors less than 1000 tags, and that each tag is monitored by at least 2 basestations.

MRT Range and Life Time: We are in the process of prototyping MRTs. Our initial MRT prototypes are 
designed to operate at a $0 \mathrm{dBm}$ power level. A jewellery store is an inherently low scattering environment with very little clutter as the merchandise is displayed for easy viewing. Even very conservative measurements made by [15] suggests that a $0 \mathrm{dBm}$ transmission can be heard at least $10 \mathrm{~dB}$ above the noise floor up to an average distance of $100 \mathrm{~m}$. $0 \mathrm{dBm}$ power can be delivered by a 5 percent efficient general purpose transceiver like [16] by drawing $20 \mathrm{~mW}$ from the battery. A transmission burst of $100 \mu$ secs causes the radio to draw an average power of $2 \mu \mathrm{W}$ or $0.6 \mu \mathrm{A}$ from a $3 \mathrm{~V}$ coin cell. A traditional low power microcontroller [17] uses $0.6 \mathrm{~mA}$ with a 1 $\mathrm{MHz}$ clock. Hence with a transmission burst of $100 \mu \mathrm{secs}$, average current drawn falls to $0.06 \mu$ amps. If we run the controller longer for additional processing, the consumption is still going to be negligible compared to the radio. A custom chip will bring this power down even more. A commercially available thin lithium battery source [18] can provide $6 \mathrm{mAh}$ at $3 \mathrm{v}$ for a $3 \mathrm{~cm}^{2}$ size. With the power drain dominated by the radio, a very conservative battery life estimate can be given at $10^{4}$ hours or 416 days. It is important to note that this value is an absolute lower bound on the lifetime since it takes into account the radio inefficiencies and considers the use of a very general purpose (non-optimal for our application) microprocessor and radio.

Tag Localization And System Wide Detection: Each reader has a set of tag ID's associated with it which are stored in its ID-table. Note that a tag ID is to be seen by more than one reader (by ensuring proper basestation density during deployment). Each reader is responsible for checking the presence of all the tags described in its IDtable. The server runs an enterprise-wide application that collects information from all the readers. The application populates a list of tags, their locations (based on the readers which see them), and a time stamp of the last reported presence from a reader. The absence of a tag from a basestation's environment causes a local event which requests the application running on the server to look for the tag's presence in the readers that are at most $\epsilon$ hops away. $\epsilon$ is dependent on the time required to reliably detect a tag at each basestation, maximum velocity of the tag in the environment, and the distance between the basestations in the grid. For example, if there is a limitation that the MRT must be carried by a human then [19] gives the maximum speed at which the MRT may move (approximately $10 \mathrm{~m} / \mathrm{sec}$ ). Consider, the distance between basestations as $5 \mathrm{~m}$ and minimum detection time per basestation as 5 seconds, $\epsilon$ may be estimated as 6 hops (an approximate perimeter of $30 \mathrm{~m}$ ). A flooding based approach may prove to be an overkill with group movements of tags.

\section{Vi. Conclusions And Future Directions}

This paper presents a feasibility study for a transmitonly tag based active RFID tracking system. We show that the receiver on the tags is a dominant but unnecessary source of system cost and power consumption. A receiver- less design, has enough entropy to permit detection of tags at a low error rate. Calculations and rigorous simulations show that the interference in a completely random system like ours requires the use of good signal processing schemes at the receiver. We show that the problem being solved here has similarity to a conventional CDMA multiuser detection problem with some fundamental differences. Our results show that, by detecting tags across multiple epochs and using a networked basestation approach, we can have low error rates with a set of 5000 tags. Future work in this direction involves improvement of the detection algorithms by employing some phase estimation schemes and development of a hardware prototype for the RollCall deployment in a jewelery store.

\section{REFERENCES}

[1] J. Wieselthier, A. Ephremides, and L. Michaels, An Exact Analysis And Performance Evaluation Of Framed Aloha With Capture, IEEE Transactions on Communications, pg 125 - 137, 1989.

[2] K. McCathie, L. Wollongong, The Pros And Cons Of RFID In Supply Chain Management, Proceedings of the International Conference on Mobile Business, pg 623 - 629, ISBN: 0-76952367-6, Jul 2005.

[3] R. Want, An Introduction To RFID Technology, IEEE conference on Pervasive Computing, 5(1), pg 25 - 33, Mar 2006.

[4] J. Armstrong, J. Richert, J. Palmer, RFID Communication Method And System, European Patent ID EP1211630, Jun 2006.

[5] R. Bridgelall, Bluetooth/802.11 Protocol Adaptation for RFID Tags, Proceedings of the 4th European Wireless Conference, Florence, Italy, Feb 2002.

[6] M. Stemm, R. Katz, Measuring And Reducing Energy Consumption Of Network Interfaces In Hand-held Devices, IEICE Transactions on Communications, vol. E80-B, no. 8, pg 1125 1131, Aug 1997.

[7] W. Ye, J. Heidemann, Wireless sensor networks, Kluwer Academic Publishers, pp. 73 -91, ISBN:1-4020-7883-8, May 2004.

[8] IEEE 802.11, 1999 Edition, IEEE Standards for Information Technology - Part 11: Wireless LAN Medium Access Control $(M A C)$ and Physical Layer (PHY) Specifications, ISO/IEC 8802 $11,1999$.

[9] N. Abramson, Development of the ALOHANET, IEEE Transactions on Information Theory, ISSN: 0018-9448, Vol. 31, No. 2, pg 119 - 123, Mar 1985.

[10] L. Kleinrock, F. Tobagi, Packet switching in radio channels: Part I - carrier sense multiple access modes and their throughput delay characteristics, IEEE Transactions on Communications, vol. 23 , no. 12 , pg 1400 - 1416, Dec 1975.

[11] S. Verdu, Multiuser Detection, Cambridge University Press, Pitt Building, Trumpington Str., Cambridge, UK, First Ed., ISBN: 0521593735, Aug 1998.

[12] A. Muqattash, M. Krunz, and W. E. Ryan, Solving the NearFar Problem in CDMA-Based Ad Hoc Networks, Elsevier Ad Hoc Networks Journal, Vol. 1, No. 4, pp. 435 - 453, Nov 2003.

[13] J. Holtzman, DS/CDMA Successive Interference Cancellation, in IEEE Int. Symp. Spread Spectrum Techniques and Applications, Oulu, Finland, pp. 69 - 78, Jul 1994.

[14] P. Patel, J. Holtzman Analysis of A DS/CDMA Successive Interference Cancellation Scheme Using Correlations, Global Telecommunications Conference, pp. 76 - 80, vol. 1, Dec 1993.

[15] D. Dobkin, Indoor Propagation Issues for Wireless LANs , RF Design, pg 40 - 46, Sep 2002.

[16] Chipcon Inc. Catalogs, Chipcon CC1100 Data Sheet, http : //www.chipcon.com/.

[17] Silicon Laboratories Catalogs, www.silabs.com/mcu/, 8051 microcontroller datasheet.

[18] Power Stream Batteries and Power Pack Catalogs, Specifications For Thin Lithium Batteries, http : //www.power stream.com/.

[19] "Running", Guinness Book of Records 1999. London: Random House, 1999. 200m: 19.32 Michael Johnson (US), Jul 1996. 